\title{
SAMPLE-RETURN MISSIONS TO THE ASTEROID EROS
}

\author{
ALFRED C. MASCY \\ NASA Ames Research Center \\ and \\ JOHN NIEHOFF \\ IIT Research Institute
}

\section{MISSION SELECTION}

Of the many asteroids that are cataloged, selection of a target planetoid was narrowed to the Mars-crossing asteroids. The main belt and Trojan asteroids are much farther away and require longer voyage times and propulsive energy to accomplish sample returns. Within the Mars-crossing group, those asteroids were surveyed that have orbits inclined less than $15^{\circ}$, perihelia within $0.2 \mathrm{AU}$ of Earth's orbit (and therefore require less propulsive energy), and diameters greater than $1 \mathrm{~km}$ (to assist terminal observation and rendezvous). Eros was chosen as representative of this class.

The mission consists of the following phases: (1) Earth departure; (2) trans-Eros trajectory; (3) Eros approach, rendezvous, site selection, topographical coverage, and docking; (4) surface operations including sample acquisition; (5) Eros departure; (6) trans-Earth trajectory; (7) Earth approach, capsule separation, and orbit capture maneuver; and (8) in-orbit quarantine until deboost command or orbit retrieval.

\section{CANDIDATE EXPERIMENTS}

A number of scientific instruments were considered in formulating a baseline Eros rendezvous/sample-return mission. These candidate instruments are listed in table I along with related science measurables, the mission phases during which they would operate, and an indication of what their mass and power requirements are expected to be. The instruments are divided into two categories: (1) those instruments essential for sample return and (2) those desirable for a viable rendezvous and landing science mission. Because not all instruments would operate simultaneously, the total power requirement is somewhat less than the accumulated one. By far the most important phases of the mission for instrument operation are stationkeeping and landing. Because the total science payload is only $90 \mathrm{~kg}$, it was assumed that all instruments presented in table I would be included in the science payload of the baseline 


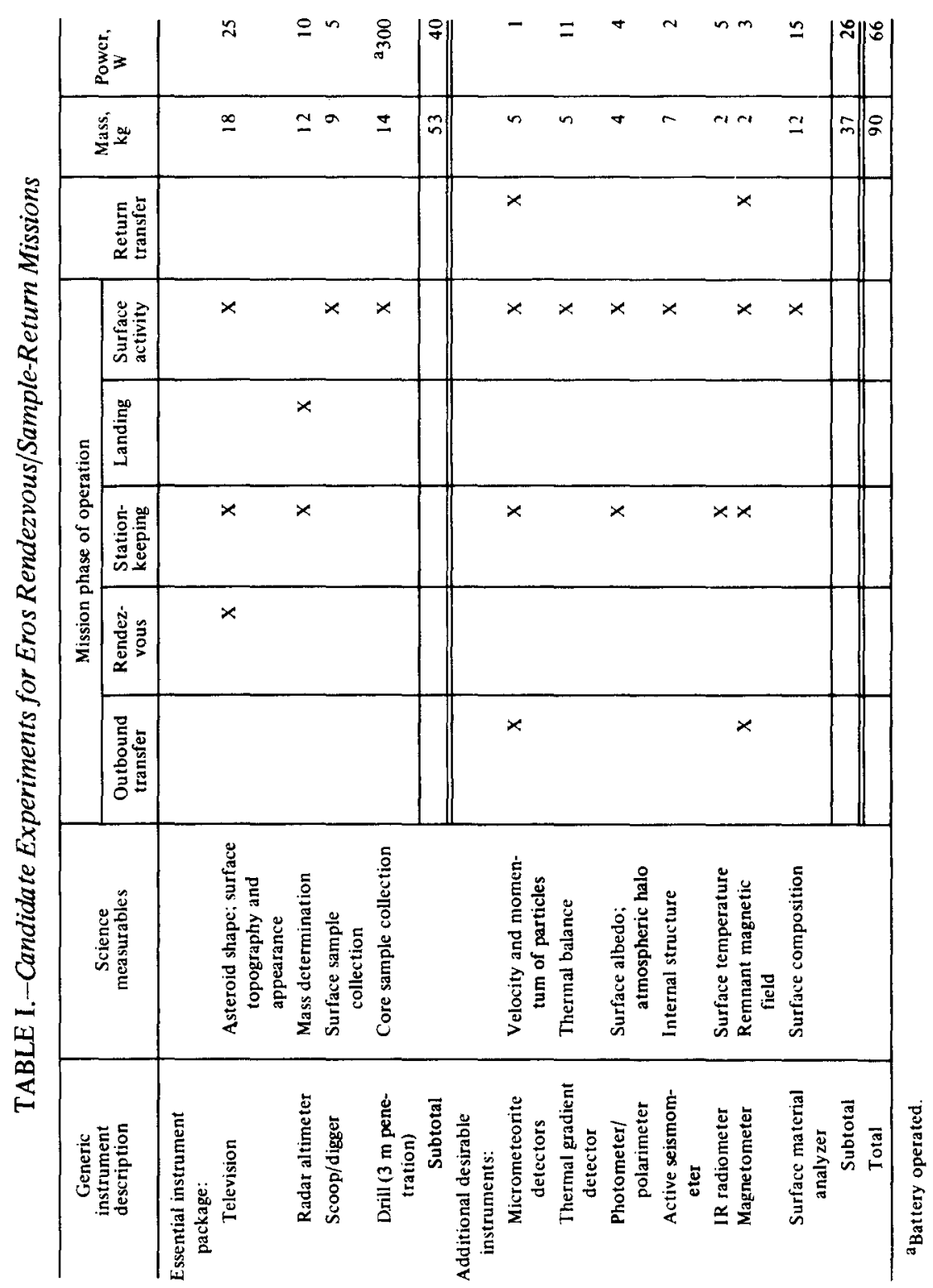


mission. These instruments are only suggested as typical of what a rendezvous/ sample-return payload might be and are not a specifically recommended set of experiments. A description of another payload complement is found in the Meissinger and Greenstadt paper. ${ }^{1}$ A mass of $150 \mathrm{~kg}$, including the $90 \mathrm{~kg}$ of science and support equipment, is assigned to the trans-Eros and asteroid surface operations. This mass is jettisoned upon departure from Eros.

\section{RENDEZVOUS OPERATIONS}

It is assumed that a number of stationkeeping maneuvers would be required following rendezvous with Eros before landing (or docking) on the asteroid. These maneuvers would achieve the necessary reconnaissance needed for landing site selection (preferably on one of the poles) and most importantly provide ample time for the remote sensing measurements indicated in table $I$ at various locations around the asteroid.

The recommended sequence of maneuvers is illustrated in figure 1. They start at point 1 , which is $1000 \mathrm{~km}$ from the asteroid in the solar direction. This position seems most compatible with rendezvous because the asteroid should be well lighted in a dark sky for optical tracking during final rendezvous. The sequence of maneuvers allows the spacecraft to approach the asteroid to within $250 \mathrm{~km}$ from three directions: Sun side, terminator, and dark side. The first two approaches should permit an accurate determination of the polar direction $^{2}$ (for landing) and provide good surface appearance data under limiting illumination conditions. The third approach would permit a search

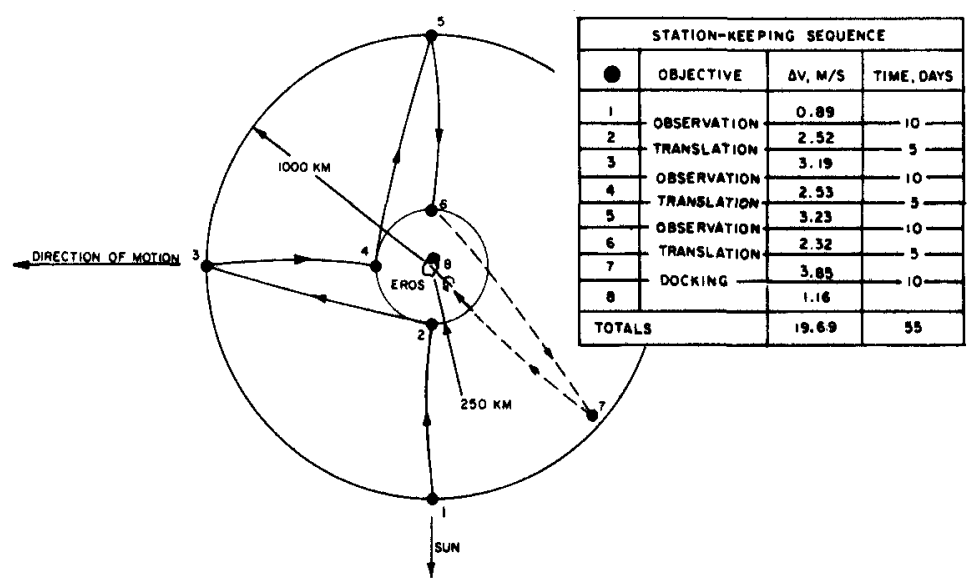

Figure 1.-Stationkeeping profile for 1977 Eros sample-return mission. Dashed line indicates out-of-plane motion.

\footnotetext{
${ }^{1}$ See p. 543.

${ }^{2}$ Editorial note: By 1975 we should know the orientation of the rotation axis to within a few degrees; see p. 133.
} 
from the dark side for a dust cloud or atmospheric halo around Eros, using photometers. The final approach is recommended along the rotation axis to landing. The entire sequence would take about 55 days as shown in the figure. Remote teleoperator landing of a spacecraft is described by Meissinger and Greenstadt; ${ }^{3}$ however, because of the large communication distance, response times are on the order of a half hour.

The stationkeeping and landing operations were regarded as essentially the same for both the ballistic and low-thrust flight modes. The weight breakdowns for the baseline mission assume that the entire spacecraft (less outbound propulsion) is docked with the asteroid during sample collection and surface experimentation. A budget of $75 \mathrm{~m} / \mathrm{s}$ has been allowed for all stationkeeping, docking, and separation maneuvers at Eros. A conservative estimate of $350 \mathrm{~kg}$ is assigned to the landing mechanism and maneuver/docking propulsion inert mass. This mass is jettisoned upon departure from Eros.

\section{PROPULSIVE ENERGY REQUIREMENTS}

The analysis of round-trip interplanetary space missions generally requires the survey of compatible outbound and return trajectories. Whereas one-way orbiter or flyby missions may utilize near-optimum outbound trajectories, round-trip missions compromise the performance on both outbound and retum legs so that the overall mission energy requirements are minimized. An expedient method of examining large quantities of round-trip trajectory

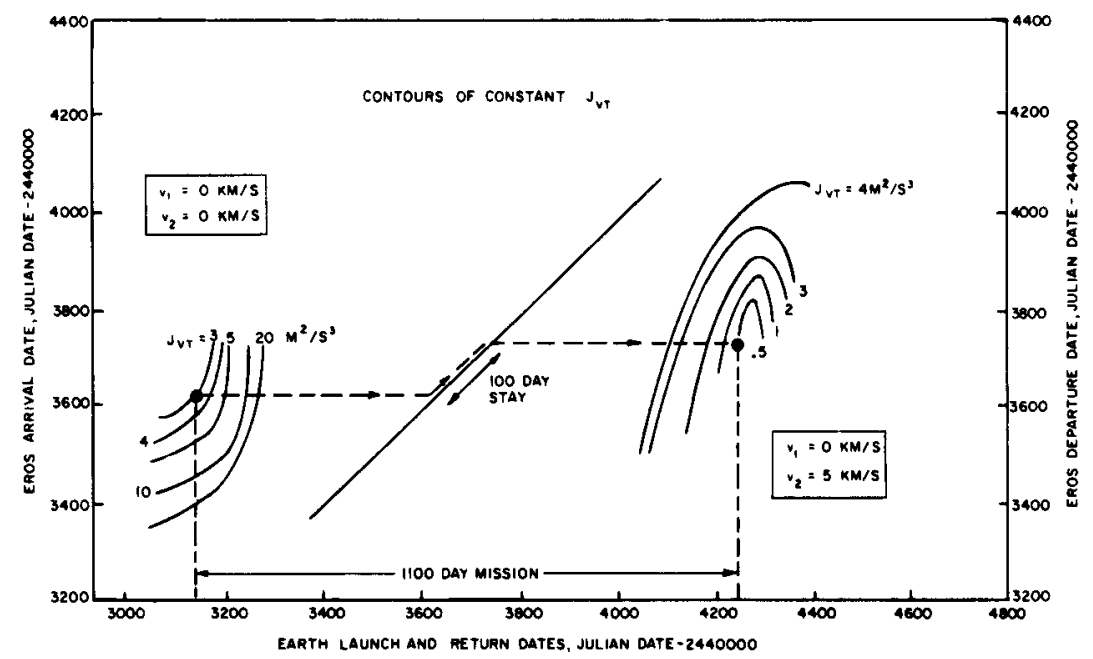

Figure 2.-Solar electric contour map for 1977 Eros sample-return mission. $v_{1}$ is the hyperbolic excess velocity at Earth departure, $v_{2}$ is the hyperbolic excess velocity at Eros arrival, and $J_{V T}$ is a measure of electric-propulsion energy requirements.

${ }^{3}$ See p. 552. 


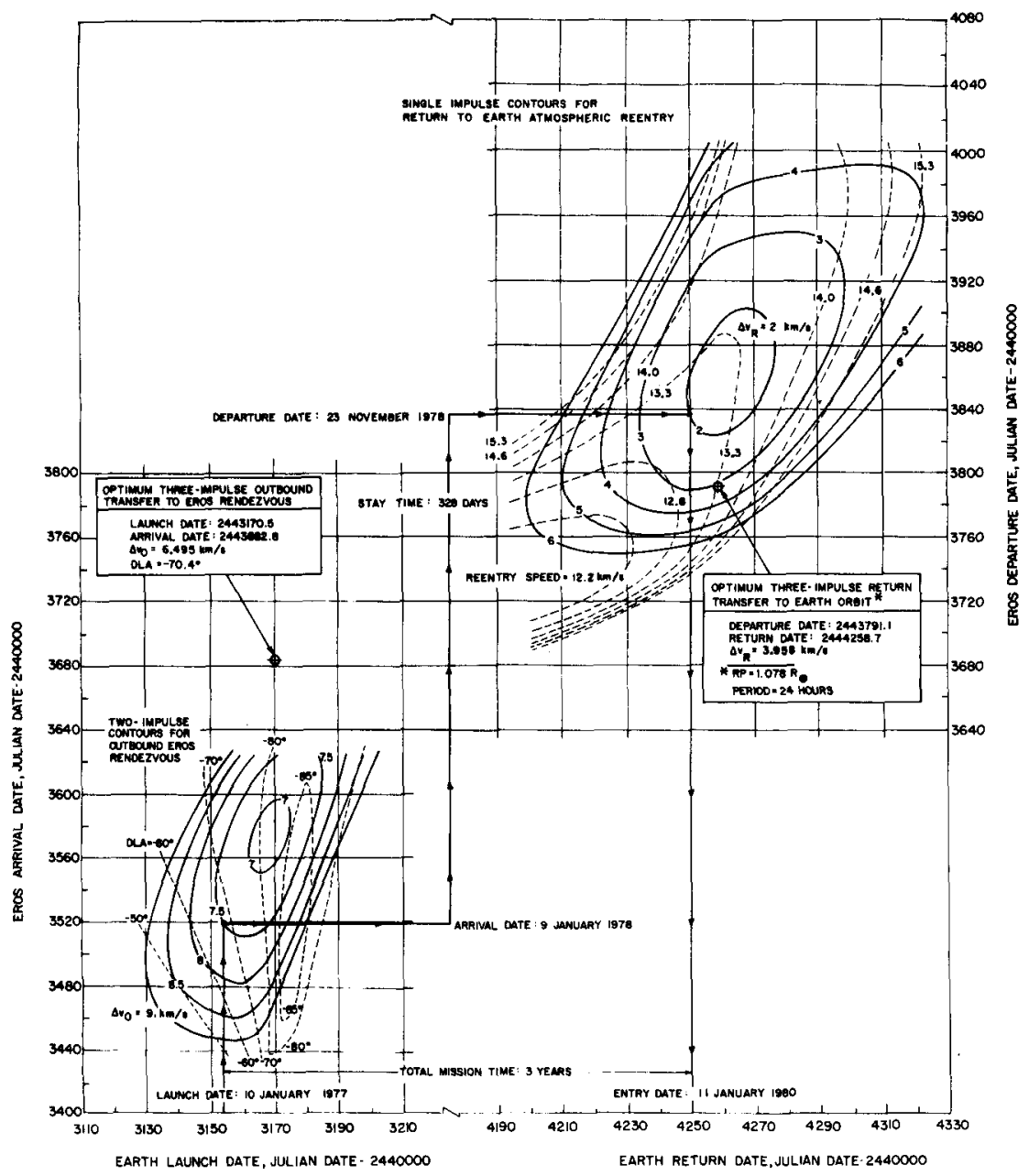

Figure 3.-Ballistic impulse contour map for 1977 Eros sample-return mission.

characteristics is shown in figures 2 and 3 for the 1977 opportunity to Eros as accomplished by an electric-propulsion flight mode and a chemical-propulsion flight mode, respectively.

\section{ELECTRIC-PROPULSION FLIGHT MODE}

A measure of the propulsive energy requirements for electrical rocket systems that employ finite-thrust trajectories is given by the time-integrated effect of the continually applied low acceleration. In figure 2, this energy measure has the symbol $J$ with units of square meter per cubic second. The higher the $J$, the more difficult the mission. Mascy, Dugan, and Pitts (1968) 
have shown that the systematic mapping of this energy parameter provides a convenient technique for determining the best launch and arrival dates and the effect of varying trip times and stay times. For both figures 2 and 3, the launch and return dates at Earth are given along the abscissa and the arrival and departure dates at Eros are given along the ordinate. An example of an 1100 day mission is shown in figure 2 departing from Earth on Julian date 2443150, arriving at Eros 2443625, staying 100 days, departing from Eros 2443725, and returning to Earth on 2444250 . In a similar manner, one can fashion missions of varying outbound or return-leg trip times, launch dates, stay times, etc.

The electric-propulsion system assumed in this report utilizes solar photovoltaic cells to convert the Sun's energy into electrical energy for acceleration of ionized mercury propellant. A power level of $10 \mathrm{~kW}$ and a thrustor specific impulse of $3000 \mathrm{~s}$ are used in the analysis. Similar to the systems and technology described in the TRW, Inc., report, the overall electric-propulsion module has a mass of $300 \mathrm{~kg}$. An additional mass of approximately $400 \mathrm{~kg}$ has been assigned to the interplanetary bus, which comprises the engineering subsystems such as communications and data handling, central computer and mission sequencer, thermal and attitude control, and support structures. The launch vehicle used to inject the solar electrically propelled interplanetary vehicle onto a trans-Eros trajectory is the Titan IIID/Burner II.

\section{CHEMICAL-PROPULSION FLIGHT MODE}

In figure 3 is presented the contours of impulsive velocity increment, which is a measure of the propulsive energy requirements for chemical rocket systems that employ ballistic trajectories. This energy measure has the symbol $\Delta v$ with the units kilometers per second. The higher the $\Delta v$, the more difficult the mission. The outbound transfer contours in the lower left give the total impulse for two maneuvers: (1) impulsive escape from a $185 \mathrm{~km}(100 \mathrm{n}$. mi.) parking orbit at Earth and (2) impulsive rendezvous at Eros. In the background, dotted contours of declination of the launch asymptote (DLA) are given. Notice that the region of minimum total outbound impulse, $\Delta v_{O}<7.5 \mathrm{~km} / \mathrm{s}$, lies over absolute values of DLA that are greater than $-70^{\circ}$. This implies a serious problem for launches from the Eastern Test Range because range safety constraints require that DLA $<36^{\circ}$. Return transfer contours of impulsive departure velocity at Eros are presented in the upper right of figure 3. Dotted curves of Earth reentry speed of the return-sample capsule are shown in the background. The region of minimum departure impulse, $\Delta v_{R}<2 \mathrm{~km} / \mathrm{s}$, lies over Earth reentry speeds of less than $13.7 \mathrm{~km} / \mathrm{s}$ $(45000 \mathrm{ft} / \mathrm{s})$, which should not pose a reentry technology problem. A sample mission that requires a total trip time of $3 \mathrm{yr}$ is laid out with the arrowed line. The stay time at Eros would be almost $1 \mathrm{yr}, 328$ days. To use outbound and return transfers that are reasonably close to minimum required energies, it can 
be observed from the figure that one is forced to accept a total trip time on the order of $3 \mathrm{yr}$.

In addition to the direct two-impulse transfers discussed thus far for ballistic systems, optimum three-impulse trajectories were also investigated for the following reasons: (1) a potential for lower total impulse, due to the $11^{\circ}$ inclination of Eros' orbit to the ecliptic; (2) the possibility of improving the launch problem due to large DLA values; and (3) the inclusion of Earth capture-orbit recovery, rather than direct reentry, as a more cautious mission profile in view of back-contamination uncertainty. Optimum three-impulse outbound and return (to Earth orbit) transfer points are included in figure 3 . The total outbound impulse $\Delta v_{O}$ is indeed less than required by the minimum two-impulse transfer, but a DLA of $-70^{\circ} .4$ does not solve the launch problem. The three-impulse return transfer to Earth orbit requires a $\Delta v_{R}$ of less than $4 \mathrm{~km} / \mathrm{s}$, which, although difficult, is not impossible to achieve with chemical propulsion.

Table II summarizes the transfer characteristics for the two most promising ballistic mission profiles for the launch opportunities of 1974, 1977, and 1979. Both profiles utilize three-impulse outbound transfers to Eros rendezvous. The first profile uses a minimum single-impulse return trajectory to Earth reentry, whereas the second profile requires a three-impulse return transfer to Earth orbit. Note that, for the multi-impulse return, the arrival impulse provides a $12 \mathrm{hr}$ Earth orbit that is more practical for subsequent recovery than the $24 \mathrm{hr}$ orbit suggested in figure 3 . Only the $12 \mathrm{hr}$ recovery orbit is considered in the remainder of this paper. The results presented in table II are quite variant. The best (lowest total impulse) outbound transfer occurs in 1977. The best launch conditions occur in 1974 when DLA $=-49^{\circ} .1$. The best return transfers occur in 1979. In 1974, the best single-impulse return trajectory to Earth reentry is worse than the three-impulse transfer ending in Earth orbit. The stay times are variable, ranging from 88 to 378 days depending upon the opportunity and return transfers selected. The one parameter that does not vary much is the total trip time, which is always very nearly $3 \mathrm{yr}$. This was observed earlier and apparently is quite stable regardless of launch opportunity or mission profile.

The launch vehicle used to inject the ballistic chemically propelled interplanetary vehicle into a trans-Eros trajectory is the Titan IIID(7)/Centaur. For the ballistic flight mode, all major impulsive velocity increments, including a $200 \mathrm{~m} / \mathrm{s}$ guidance requirement, are provided by a space-storable chemicalpropulsion system that has an assumed specific impulse of $385 \mathrm{~s}$. An interplanetary bus has been assumed for the chemical flight mode that has a mass of $250 \mathrm{~kg}$ and comprises the engineering subsystems.

Upon return to Earth, both the electric- and chemical-propulsion flight modes assume a small solid rocket engine to provide the required velocity increment to place the return capsule (containing the sample) into orbit. Characteristics of the final orbit are: $500 \mathrm{~km}$ periapsis altitude, $40000 \mathrm{~km}$ apoapsis altitude, and $12 \mathrm{hr}$ period. 


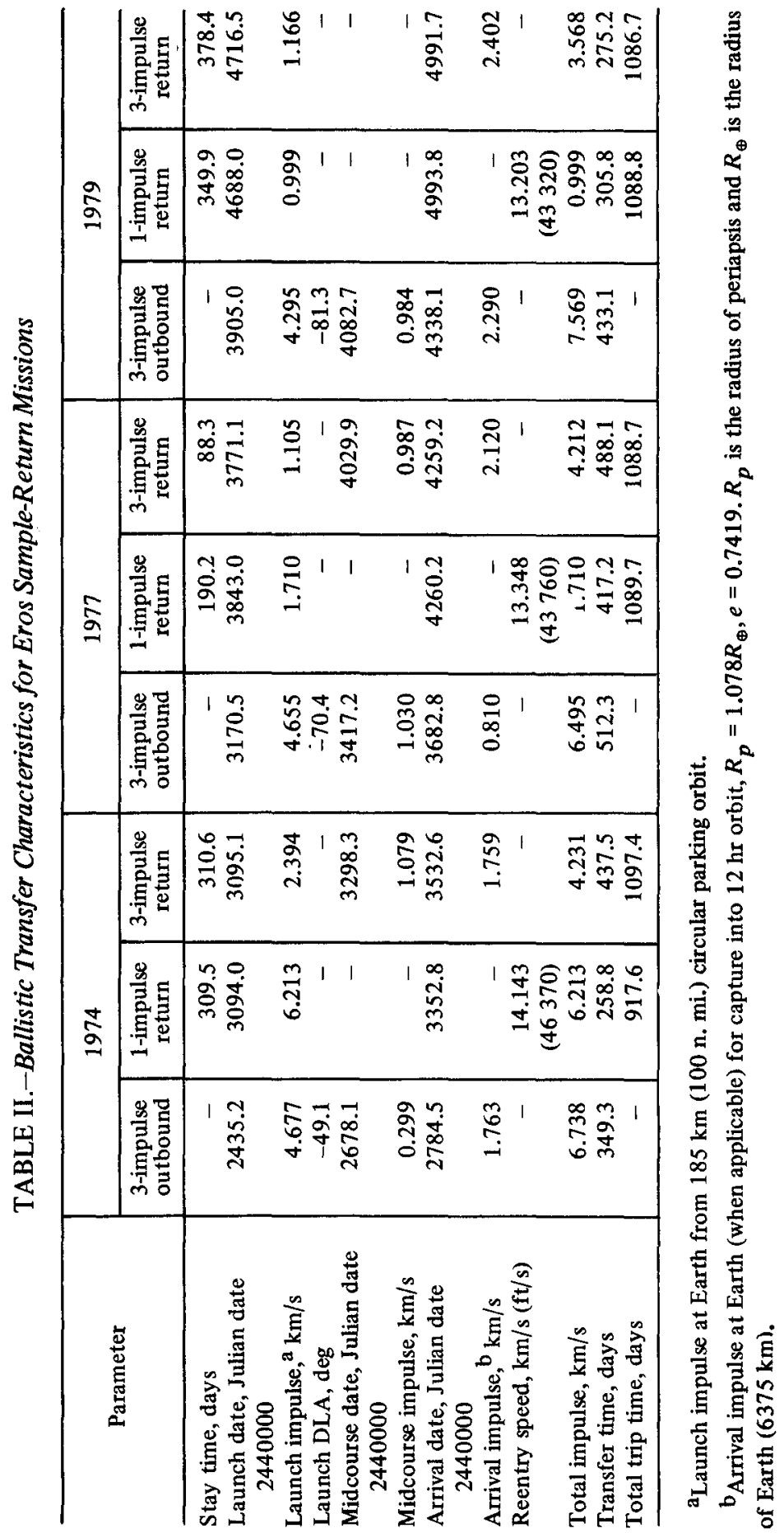




\section{Effect of Trip Time}

\section{MISSION RESULTS}

With the assumptions made earlier, round-trip missions of various overall times were investigated for an Earth launch opportunity occurring during 1977. The sensitivity to trip time of capsule mass returned to Earth orbit is shown in figure 4 for the solar electric flight mode. There is a strong effect of overall time on the Eros round-trip mission with the maximum returned mass of $260 \mathrm{~kg}$ obtained for a time of 1100 days or approximately $3 \mathrm{yr}$. Capsule mass decreases very rapidly for trip times much shorter or longer than the optimum. The same characteristic has been found for the chemical flight mode.

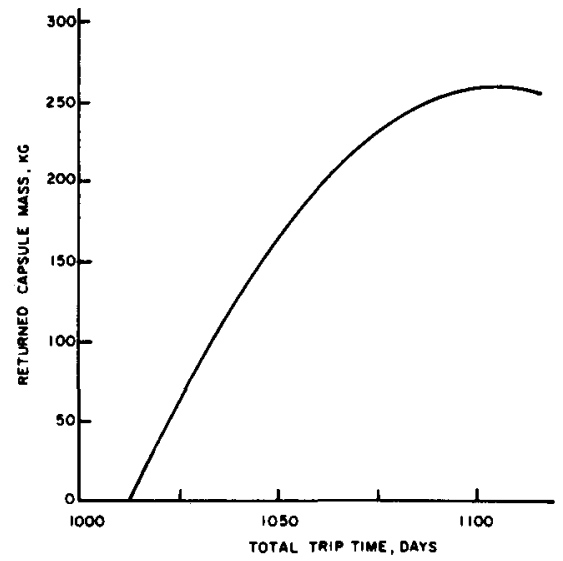

Figure 4.-Trip-time effect on returned capsule mass. Solar electric flight mode; Titan IIID/Burner II; 1977 launch opportunity; stay time $=50$ days; Earth return orbit $=500 \times 40000 \mathrm{~km}$.

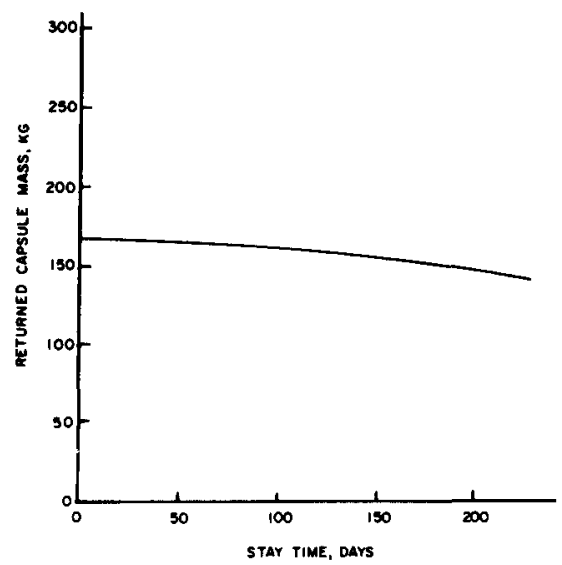

Figure 5.-Stay-time effect on returned capsule mass. Solar electric flight mode; Titan IIID/Burner II; 1977 launch opportunity; trip time $=1050$ days; Earth return orbit $=500 \times 40000 \mathrm{~km}$.

\section{Effect of Stay Time}

Stay time is defined as the total time spent in the vicinity of Eros. It includes the rendezvous and stationkeeping maneuvers, topographical survey, surface operations, and departure maneuvers. An investigation of the effect of stay time on returned mass is shown in figure 5 for the solar electric flight mode. Over a range of stay times, there does not appear to be any significant effect on the capsule mass. This allows the freedom to perform whatever operations in the vicinity of Eros are desired. Additionally, it was found that the optimum total trip time remains relatively constant at $3 \mathrm{yr}$ as stay time is varied. That is, trip time is not appreciably shortened or lengthened as stay time is varied. 


\section{Effect of Launch Year Opportunity}

In addition to the 1977 launch opportunity discussed thus far, other launch years were surveyed. Opportunities for round-trip missions to Eros occur about $2 \mathrm{yr}$ apart, and their effect on returned mass is shown in figure 6 . The open bars relate to the return capsule mass in Earth orbit and the shaded bars are merely an estimate of the soil sample size contained within the capsule. A scaling law used on a recent Mars sample-return study (Friedlander, 1970) relates the sample mass $m_{s}$ to the return capsule mass $m_{c}$ by the relationship

$$
m_{s}=0.44\left(m_{c}-29.5\right)
$$

In figure $6(a)$ is shown the ballistic flight mode. For the years surveyed, the launch opportunity in 1977 results in the maximum sample returned. Similarly, for the solar electric flight mode shown in figure 6(b), the year 1977 is the most favorable opportunity for a sample return from Eros. Preceding and succeeding years have decreasing return mass. A preliminary estimate of the synodic cycle is $16 \mathrm{yr}$, at which time (1993) a most favorable opportunity should occur again.
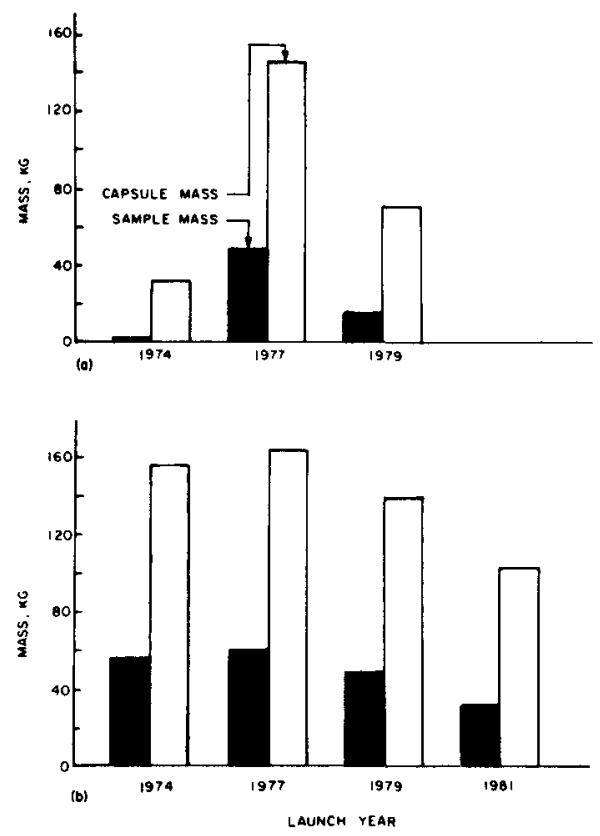

Figure 6.-Launch opportunity effect on returned mass. (a) Ballistic flight mode; Titan IIID(7)/Centaur $/ 385$; total trip time $=3 \mathrm{yr}$; stay time is variable (88 to 378 days); Earth return orbit $=500 \times 40000 \mathrm{~km}$. (b) Solar electric flight mode; Titan IIID/Burner II; total trip time $=3 \mathrm{yr}$; stay time $=50$ days. 


\section{TRAJECTORY GEOMETRY}

Ecliptic projections of the outbound and return trajectories for the 1977 Eros round-trip mission are shown in figure $7(a)$ for the solar electric flight mode and in figure $7(b)$ for the ballistic flight mode. Both outbound and inbound trajectories for both modes have approximately $300^{\circ}$ of travel angle. Launch dates from Earth are in early 1977. Arrival dates at Eros are at about mid-June 1978 at which time Earth is $2.2 \mathrm{AU}$ from Eros, almost directly behind the Sun. After a stay time at Eros, departure takes place in latter September with Earth still about $2.2 \mathrm{AU}$ away and very close to the sunline. Return dates back at Earth are approximately mid-January 1980, $3 \mathrm{yr}$ after launch. In table III is given a flight plan that details the events, dates, and

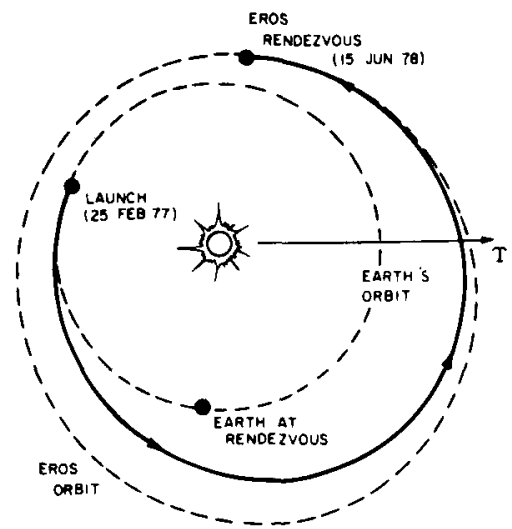

(a) EARTH TO EROS, 475 DAYS

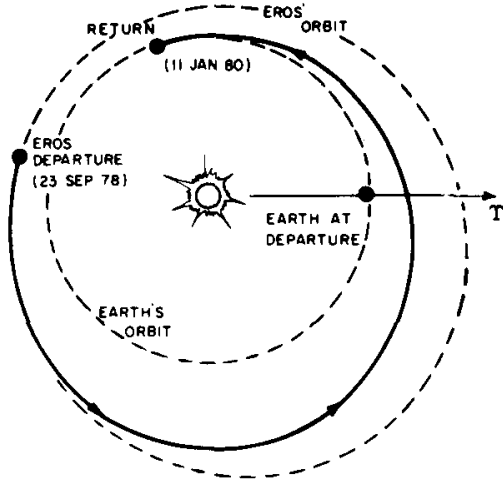

EROS TO EARTH, 475 DAYS

STAY TIME $=100$ DAYS

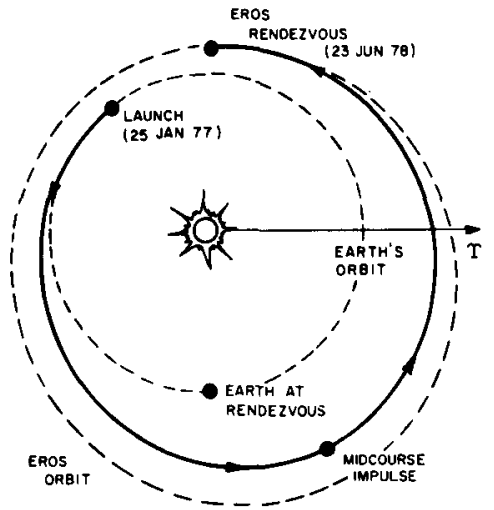

(b)

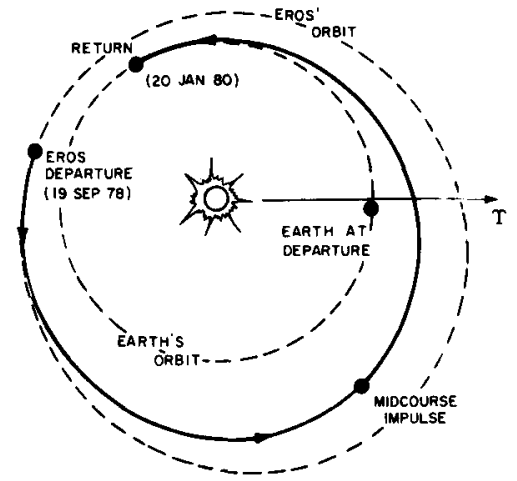

EROS TO EARTH, 488 OAYS

STAY TIME $=88$ DAYS

Figure 7.-Transfer profiles for a 3 yr Eros sample-return mission. $\Upsilon$ indicates the vernal equinox. (a) Solar electric; stay time $=100$ days. $(b)$ Ballistic; stay time $=88$ days. 


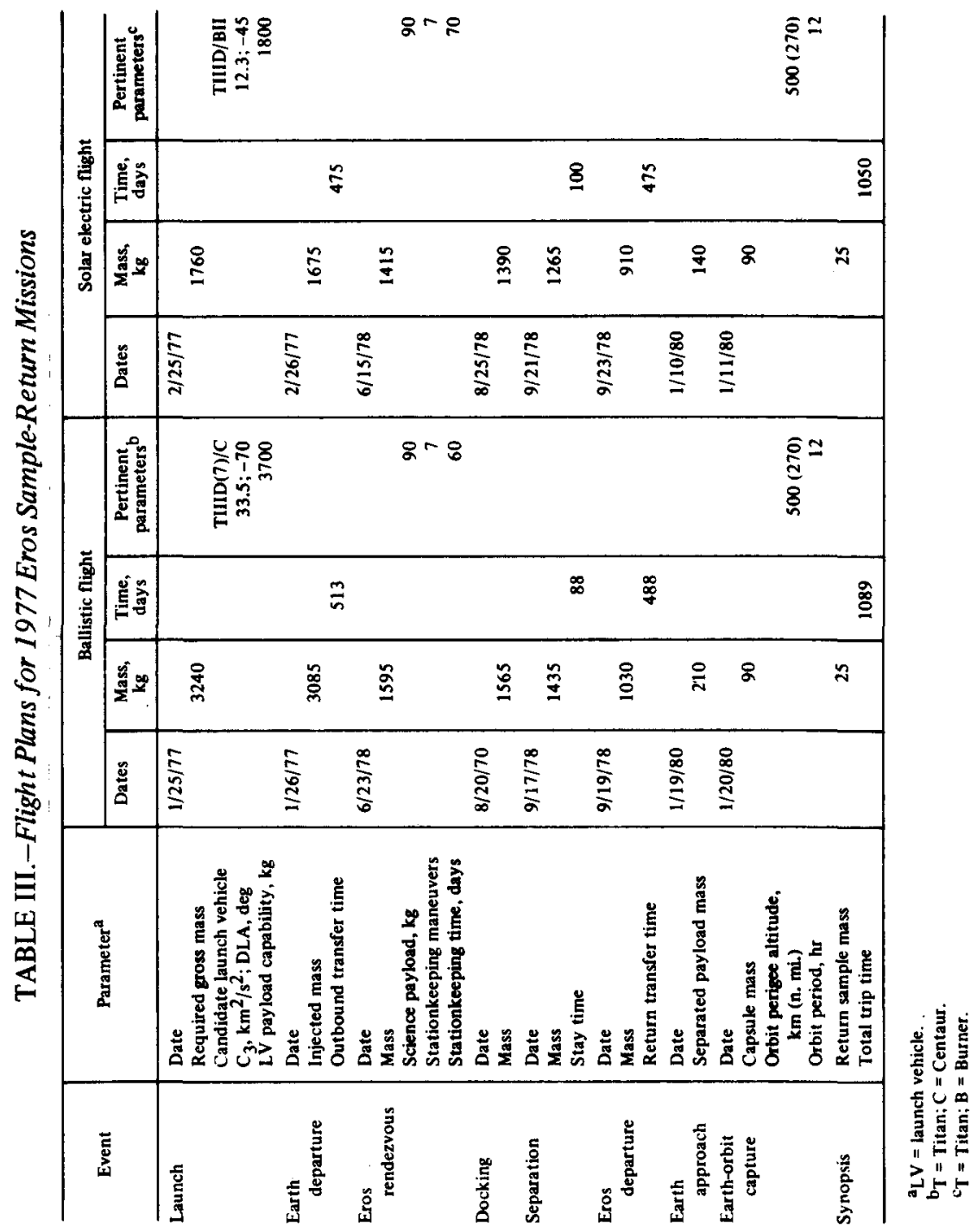


masses for the 1977 Eros sample-return mission. A fixed sample size of $25 \mathrm{~kg}$ was assumed for each flight mode. Provisions were made in the launch vehicle capabilities to provide for a nominal DLA less than $36^{\circ}$.

\section{SOLAR INTERFERENCE POTENTIAL}

It is observed from the solar electric and ballistic transfer profile graphs (figs. 7(a) and 7(b), respectively) that Earth is nearly opposite Eros, on the opposite side of the Sun, during rendezvous, stationkeeping, and docking with the asteroid. The question of whether the Sun will interfere with the necessary spacecraft/Earth communications link during these critical maneuvers was investigated. Current Deep-Space Network (DSN) communications capability requires that the Earth/spacecraft line of sight be at least $2^{\circ}$ off the Earth/Sun line (Douglas Aircraft Corp., 1965). In figure 8, the Earth/Eros line-of-sight trajectory during the 1978 Eros encounter is shown projected in a plane normal to the Earth/Sun line, positioned at the Sun. Dots are placed along this trajectory at 15 day intervals. The point of maximum solar interference $\left(3^{\circ}\right.$ separation between Eros and the Sun as seen from Earth) occurs on Julian date 2443710 (July 20, 1978). The arrival and departure points for the ballistic and solar electric baseline mission profiles are also shown on the trajectory. In both cases, these points bracket the maximum solar interference date. Fortunately, this interference $\left(3^{\circ}\right)$ appears acceptable for reliable DSN communications. Further study of this problem is needed using more recent elements of Eros' orbit to accurately determine its line-of-sight trajectory. If further communication degradation were to result, it would be necessary to shift the entire stay time by 50 to 100 days. This, of course, would have an effect on the energy requirements and payload capability of the sample-return mission.

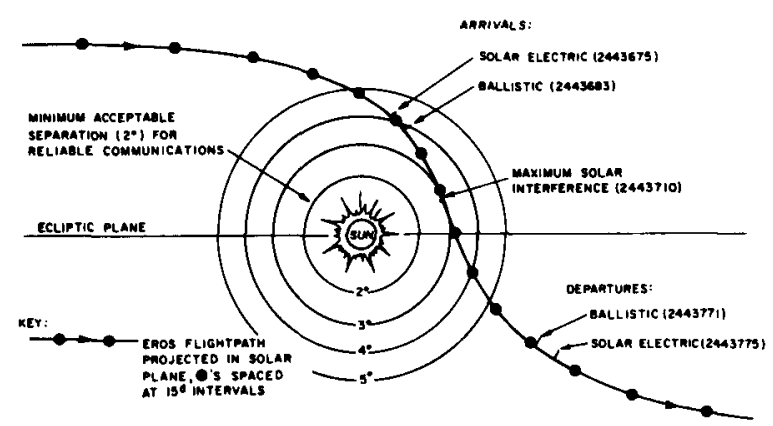

Figure 8.-Solar interference potential during 1978 Eros rendezvous.

\section{CONCLUDING REMARKS}

On the basis of the largest amount of sample material returned to Earth orbit, the 1977 launch opportunity appears to be the most favorable during a 
synodic cycle of approximately $16 \mathrm{yr}$. Mission times are invariant and are approximately $3 \mathrm{yr}$ in length. Lengthy stay times do not decrease significantly the amount of sample returned. A sample size of $25 \mathrm{~kg}$ may be returned in this mission by either a Titan IID/Burner II with a $10 \mathrm{~kW}$ solar electrically propelled vehicle or a Titan IIID(7)/Centaur/385 chemically propelled vehicle. Severe range safety problems exist (DLA $=-70^{\circ}$ ) for the ballistic flight mode. This launch problem is diminished through the use of the solar-powered flight mode. Communications between Eros and Earth during the 1978 rendezvous may be somewhat impaired because of solar interference.

Although this paper has considered round-trip sample-return missions to the asteroid Eros, many of the mission characteristics and results are applicable to other asteroids in the Mars-crossing group, such as Geographos, Apollo, Toro, and Amor. It was noted that launch opportunities to Eros occur in 1977, 1979, etc. Preliminary investigations indicate that launch opportunities to Geographos occur during the alternate years; i.e., 1976, 1978, etc. Missions to this asteroid also require $3 \mathrm{yr}$ to complete a sample return.

\section{REFERENCES}

Friedlander, Alan L. 1970, Solar Electric Propulsion Capabilities for Mars Surface Sample Return Missions. Internal Document, Illinois Inst. Technol. Res. Inst. Chicago.

Mascy, Alfred C., Dugan, Duane W., and Pitts, Samuel W. 1968, Applications of Combined Electric, High-Thrust Propulsion Systems. J. Spacecr. Rockets 5(7), 785-791.

Douglas Aircraft Corp. 1965, Study of Conjunction Class Manned Mars Trips-Part II. NASA CR 64119.

TRW, Inc. Study of a Common Solar-Electric Propulsion System for High-Energy Unmanned Missions. Contract NAS2-6040 for NASA Advanced Concepts and Missions Div., Moffett Field, Calif. 